\title{
Stiff equation of state for a holographic nuclear matter as instanton gas
}

\author{
Kazuo Ghoroku $\odot,{ }^{1, *}$ Kouji Kashiwa, ${ }^{1, \dagger}$ Yoshimasa Nakano $\odot,{ }^{\dagger}$ Motoi Tachibana, ${ }^{2, \S}$ and Fumihiko Toyoda ${ }^{3, \|}$ \\ ${ }^{1}$ Fukuoka Institute of Technology, Wajiro, Fukuoka 811-0295, Japan \\ ${ }^{2}$ Department of Physics, Saga University, Saga 840-8502, Japan \\ ${ }^{3}$ Faculty of Humanity-Oriented Science and Engineering, Kinki University, Iizuka 820-8555, Japan
}

(Received 4 August 2021; accepted 8 November 2021; published 1 December 2021)

\begin{abstract}
In a holographic model, which was used to investigate the color superconducting phase of QCD, a dilute gas of instantons is introduced to study the nuclear matter. The free energy of the nuclear matter is computed as a function of the baryon chemical potential in the probe approximation. Then the equation of state is obtained at low temperature. Using the equation of state for the nuclear matter, the TolmanOppenheimer-Volkov equations for a cold compact star are solved. We find the mass-radius relation of the star, which is similar to the one for the quark star. This similarity implies that the instanton gas given here is a kind of self-bound matter.
\end{abstract}

DOI: $10.1103 /$ PhysRevD.104.126002

\section{INTRODUCTION}

Many analyses of the holographic quantum chromodynamics (QCD) imply a schematic phase diagram shown by Fig. 1, where $\mu$ and $T$ denote the chemical potential of the quark and the temperature of the system, respectively. The solid curve represents the confinement/deconfinement transition, which is obtained as the Hawking-Page transition points from the anti-de Sitter (AdS)-soliton to the Reissner-Nordstrom (RN) background configurations through the following bulk action

$$
S_{\text {Bulk }}=\int d^{d+1} x \sqrt{-g}\left\{\mathcal{R}+\frac{d(d-1)}{L^{2}}-\frac{1}{4} F^{2}\right\},
$$

for $d=5[1,2]$. This bulk action is written by the EinsteinHilbert action with a negative cosmological constant, $d(d-1) / L^{2}$, and $(d+1)$-dimensional $U(1)$ gauge field, $F^{2} \equiv F_{\mu \nu} F^{\mu \nu}$. The first gravitational part is proposed as a model dual to the Yang-Mills (YM) theory with stronglyinteracting flavor fermions, and the gauge-field part is dual to the baryon-number current.

We notice that, for the bulk background of $d=5$, the dimension of the boundary spacetime is effectively $(3+1)$

\footnotetext{
gouroku@fit.ac.jp

†kashiwa@fit.ac.jp

¥ynakano@kyudai.jp

\$motoi@cc.saga-u.ac.jp

"f1toyoda@jcom.home.ne.jp
}

Published by the American Physical Society under the terms of the Creative Commons Attribution 4.0 International license. Further distribution of this work must maintain attribution to the author(s) and the published article's title, journal citation, and DOI. Funded by SCOAP . since one space dimension is compactified by the SherkSchwarz compactification. The above action has been first used to examine the electric superconductivity for $d=3$ [3-7] and theory with $R$-symmetry for any $d$ [8].

Recently, Eq. (1.1) has been used as a bottom-up holographic QCD model to study color superconductivity $[1,2,9,10]$ in the region $(C)$ of Fig. 1 . The analysis has been performed under the assumption that the $U(1)$ gauge field is dual to the baryon number current. The model is extended up to small $N_{c}$, where $N_{c}$ is the number of colors and the authors of Ref. [2] pointed out that the color superconducting (CSC) phase could not be found for $N_{c} \geq 2$. However, the existence of the CSC phase has been pointed out when the model is improved by adding the higher-derivative terms, the Gauss-Bonnet term, to Eq. (1.1) [10].

Another point to be noticed is the dotted line shown in Fig. 1. In the confined phase (A), baryons are constructed from $N_{c}$ quarks. Then, at a certain chemical potential, they are assembled as nuclear matter. The region (B) is called the nuclear matter phase. In previous studies, this phase has been foreseen in terms of the top-down model [11-13], based on the Sakai-Sugimoto D4/D8 model [14,15]. In these models, the baryon is introduced as an instanton which is a soliton constructed from the flavored vector mesons.

Here, based on our bottom-up holographic model (1.1), we study the nuclear matter according to the idea that it is constructed from the instanton gas. In order to provide baryons, we add the $S U\left(N_{f}\right)$ gauge-field sector to Eq. (1.1) [11,14-16], where $N_{f}$ is the number of flavors. Then the dilute gas of instantons is examined at finite chemical potential and at low temperature with the Chern-Simons (CS) term which connects the instantons and the $U(1)$ gauge field [15]. From the model of the nuclear matter 


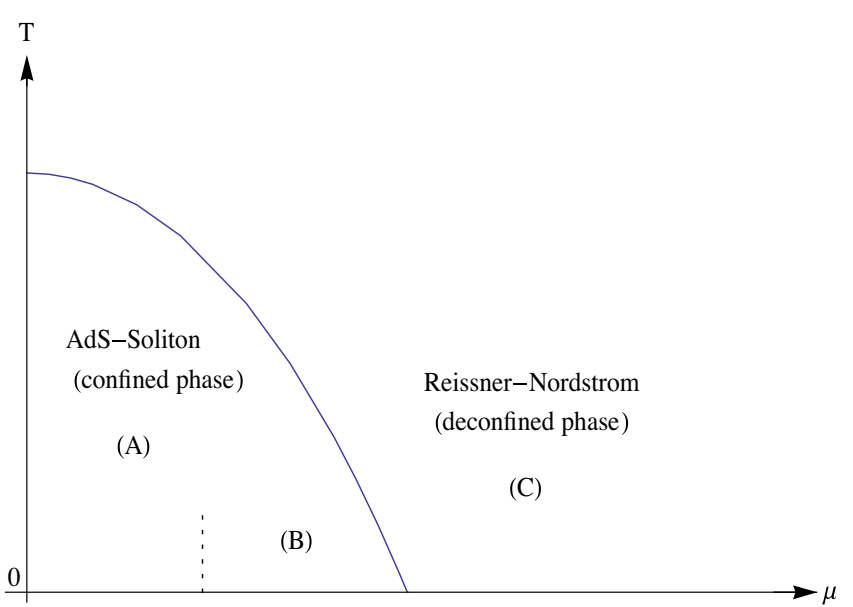

FIG. 1. A schematic phase diagram of QCD in $(\mu, T)$ plane.

given here, we can derive the equation of state (EoS). Then it can be applied to the compact cold star (like the neutron star) to estimate the relation of the mass and the radius.

For the deconfined phase (C), up to now, some holographic models have been applied to investigate the neutron star with a quark matter core [17-19]. On the other hand, the holographic investigation of the matter in the region (B) has not been performed well to study the neutron star, particularly for the mass-radius relation. Of course, several investigations for baryons have been done with the holographic models; see Refs. [20-25] for examples of previous studies.

In our approach, instead of solving the equations of motion, the flavored gauge fields are replaced by the instanton solution given in $R^{4}$ flat space with an instanton of size $\rho$. The solution is regarded as a trial function of the solutions of their equations of motion. Since, in our case, the instanton is embedded in a deformed 4D space of the curved 6D background spacetime, then the size of $\rho$ is not arbitrary, and it should be fixed at a suitable value $[2,15]$. This value is determined here by minimizing the free energy of the system at $\rho=\rho_{\min }$. Then the other physical quantities (the free energy, the chemical potential, and others) are obtained by using this $\rho_{\min }$ and other parameters of the theory. As a result, the resultant free energy can be expressed as a function of $\mu$ [2]. Then, through this approach, we can obtain the EoS of the instanton gas; namely, the nuclear matter.

Another quantity which characterizes the instanton gas is the speed of sound $\left(C_{s}\right)$ of the system. We find the condition of its value as $1 / 2<C_{s}^{2}<1$; although it preserves the causality since it is smaller than unity, the lower bound is $1 / 2$, which is large compared to that of the nuclear matter.

In this article, $S U(2)$ flavored vector fields are introduced. And the instanton-type soliton solution of the vector fields is considered as the baryon, which can be observed in the confined region as shown in Fig. 1. In the confined phase, the CSC phase is realized for $\mu>4.7$ [2]. However, when the backreaction is considered as in the present case, this CSC phase region is replaced by the newly appeared $\mathrm{RN}$ deconfined phase, which becomes dominant for $\mu>$ 1.7 [2]. Since we are considering the baryons in the confined phase, we avoid discussing the EoS in CSC phase.

In the next section, our bottom-up model is proposed by introducing a dilute gas of instantons. In Sec. III, we explain how to embed the instantons in the confined background metric. In Sec. IV the free energy of the system is computed according to our method, and EoS for the instanton gas is obtained at low temperature. In Sec. V, using the EoS, the Tolman-Oppenheimer-Volkov (TOV) equations for a compact star are solved to find the massradius $(M-R)$ relation. In Sec. VI, our results are compared with the ones from nuclear physics and astronomical observations. In the final section, the summary and discussions are given.

\section{BOTTOM-UP CSC MODEL WITH NUCLEAR MATTER}

We start from the bottom-up holographic model which is proposed as Yang-Mills theory with a color superconducting phase. It is given by the following gravitational theory [2-4].

$$
\begin{gathered}
S=\int d^{d+1} \xi \sqrt{-g} \mathcal{L} \\
\mathcal{L}=\mathcal{L}_{\text {Gravity }}+\mathcal{L}_{\mathrm{CSC}} \\
\mathcal{L}_{\text {Gravity }}=\mathcal{R}+\frac{d(d-1)}{L^{2}} \\
\mathcal{L}_{\mathrm{CSC}}=-\frac{1}{4} F^{2}-\left|D_{\mu} \psi\right|^{2}-m^{2}|\psi|^{2} \\
F_{\mu \nu}=\partial_{\mu} A_{\nu}-\partial_{\nu} A_{\mu}, \quad D_{\mu} \psi=\left(\partial_{\mu}-i q A_{\mu}\right) \psi .
\end{gathered}
$$

This describes $(d+1)$-dimensional gravity coupled to a $U(1)$ gauge field, $A_{\mu}$, and a charged scalar field, $\psi$. Here we consider the case of $d=5$. The charge $q$ denotes the baryon number of the scalar $\psi$, and it is chosen as $q=2 / N_{c}$ to represent the quark-Cooper pair formation. The mass $m$ is given to reproduce the corresponding conformal dimension of the diquark operator dual to the scalar field $\psi$ with the charge $q=2 / N_{c}$. Here, we put $1 / 2 \kappa_{6}^{2}=1$, and the dimensionful $U(1)$ gauge-coupling constant is set as unit. So, the metric is dimensionless and $A_{\mu}$ has dimension one as in the $(3+1)$-dimensional case.

Previously, the above holographic model, has been considered to be dual to the superconductor of the electric charge [3,4] and of the $R$ charge [6], and it was recently extended to a theory dual of the color superconductor in $[1,2,9]$. 
Here we add the sector of the $S U\left(N_{f}\right)$ gauge fields, which is used to build the nuclear matter through the instanton configuration, which generates the baryon number in QCD. Here, the instanton configuration is deformed since it is embedded in a higher- dimensional curved space.

$$
\begin{gathered}
\mathcal{L}=\mathcal{L}_{\text {Gravity }}+\mathcal{L}_{\mathrm{CSC}}+\mathcal{L}_{\mathrm{V}}, \\
\mathcal{L}_{\mathrm{V}}=-\frac{1}{4} \operatorname{tr} F_{S U\left(N_{f}\right)}^{2},
\end{gathered}
$$

where $F_{S U\left(N_{f}\right)}^{2}$ denotes the two-form $S U\left(N_{f}\right)$ gauge field. The coupling constant $g_{\mathrm{YM}}^{2}$ is set as one. The mass dimension of the gauge field is taken to be one. Then, in order to pick up the coupling of the baryon number current and the instanton configuration, we add the CS term,

$$
S=\int d^{d+1} x \sqrt{-g} \mathcal{L}+S_{\mathrm{CS}}
$$

We notice that there are two ways to proceed with the analysis based on this model since both the matter and the gravity fields are living in the same 6D spacetime. Therefore, the matter fields are not confined to special $D$-branes:

(i) So, in one way, we can solve the equations of motion of all fields, gauge fields, and the gravity, in the same way. In this case, the backreaction of the gauge fields are included in the metric.

(ii) On the other hand, it is possible to restrict our calculation to the probe approximation where the equations of motion of the matter fields are solved on a fixed background given by the solution of the Einstein equations of $\mathcal{L}_{\text {Gravity }}$. This approximation is assured for the large gauge couplings.

Hereafter, we proceed the analysis according to the probe approximation (ii). The matter parts are considered as the probe of the system. So, first we fix the gravitational background by solving $\mathcal{L}_{\text {Gravity }}$.

\section{A. Instanton in $R^{4}$}

Before solving the equation of motion of our model, the ansatz for the $U(1)$ gauge field and the instanton configurations for $S U(2)$ gauge fields are given. The coordinates are denoted as $\left(\xi^{0}, \xi^{1}, \xi^{2}, \xi^{3}, \xi^{4}, \xi^{5}\right)=\left(x^{0}, x^{1}, x^{2}, x^{3}, w, z\right)$. Then, we set the ansatz for $U(1)$ gauge field as

$$
A_{b}=A_{b}(z) \delta_{b}^{0}
$$

The $S U(2)$ vector fields are set as

$$
\vec{f}_{1}=F_{a b}^{i} \tau_{i},
$$

and the following ansatz are imposed,

$$
\begin{gathered}
\left(\vec{f}_{1}\right)_{i j}=Q\left(x^{m}-a^{m}, \rho\right) \epsilon_{i j k} \tau^{k}, \\
\left(\vec{f}_{1}\right)_{i z}=Q\left(x^{m}-a^{m}, \rho\right) \tau^{i},
\end{gathered}
$$

where $\rho\left(a^{m}\right)$ denotes the instanton size (position), $\epsilon_{123 z}=1, i, j=1,2,3$, and $m=1, \ldots, 4$, where $x^{4}=z$. Then the $S U\left(N_{f}\right)$ vector part (2.7) is given for $N_{f}=2$ as

$$
\mathcal{L}_{\mathrm{V}}=-\frac{3}{2} Q^{2}\left[\left(g^{11}\right)^{2}+g^{11} g^{z z}\right] .
$$

In order to see the baryon, $Q$ is given as an instanton solution in flat $4 \mathrm{D}$ space $\left\{x^{m}\right\}$ [15],

$$
Q=\frac{2 \rho^{2}}{\left(\left(x^{m}-a^{m}\right)^{2}+\rho^{2}\right)^{2}} .
$$

However, in general, this is not a solution of our bottom-up model since this configuration is embedded in a curved space. So, here we introduce it as a trial function which is supposed to be a solution of the system under an appropriate condition. The trial function is given in our formulation such that the size parameter $\rho$ of the instanton configuration is determined to satisfy the variational principle of the energy density in our bottom-up model. We determine it by minimizing the embedded instanton energy as in Refs. [13,15]. We notice that there is an another method giving $Q$-including its $z$ dependence-by solving the embedded equations of motion [12]. Here we solve only for the size parameter.

According to the above strategy, we study the multibaryon state by replacing $Q(z)$ by the multi-instanton form with the dilute gas approximation. Then we rewrite $Q$ as

$$
Q^{2}=\sum_{i}^{N_{\mathrm{I}}} \frac{4 \rho^{4}}{\left(\left(x^{m}-a_{i}^{m}\right)^{2}+\rho^{2}\right)^{4}},
$$

where the overlap among the instantons are suppressed in obtaining $Q^{2}$. This point is checked for our solutions.

In the case of the flat space in $R^{4}$, we find the energy density as a sum of each single instanton,

$$
\int d^{4} \xi^{m} Q^{2}=2 N_{\mathrm{I}} \int_{0}^{\infty} d z \bar{q}(z)^{2}=\frac{2 \pi^{2}}{3} N_{\mathrm{I}},
$$

where

$$
\bar{q}^{2}=\frac{\pi^{2} \rho^{4}}{2\left(z^{2}+\rho^{2}\right)^{5 / 2}},
$$

for the case of a dilute gas, where the interactions between instantons are neglected. Then the result is given by one instanton "mass" times its number, $N_{\mathrm{I}}$.

In the present case, the instantons are embedded in a curved space. The metrics are functions of the coordinate $z$. 
This implies that the state of the baryon is affected dynamically by the gluons. In other words, the baryon is stable when it is embedded in the background corresponding to the confined phase. Furthermore, these baryons would construct a nuclear matter like the neutron star as we see below.

\section{LOW TEMPERATURE CONFINED PHASE AND THE FOURTH COORDINATE $z$}

We suppose the nuclear matter would be the gas of instantons made up of the $S U(2)$ gauge fields in the confined phase. The spacetime background solution, which is dual to the low temperature confined phase, is given by $\mathcal{L}_{\text {Gravity }}$ at low temperature as the AdS-soliton solution. It is obtained as

$$
d s^{2}=r^{2}\left(\eta_{\mu \nu} d x^{\mu} d x^{\nu}+f(r) d w^{2}\right)+\frac{d r^{2}}{r^{2} f(r)},
$$

where

$$
f(r)=1-\left(\frac{r_{0}}{r}\right)^{5}, \quad r_{0}=\frac{2}{5 R_{w}},
$$

and $2 \pi R_{w}$ denotes the compactified length of $w$, which denotes the coordinate of the compact $S^{1}$. We notice that this solution is also a backreacted one when the $S U(2)$ gauge fields are set to be zero. In this case, the phase diagram in the $\mu-T$ plane is given in Ref. [2].

Here we restrict the region of the parameters, $T$ and $\mu$, to the confined phase. In fact, under the configuration (3.1), one finds a linear potential between the quark and antiquark by evaluating the Wilson loop [26]. Hence the vacuum stays in the confined phase, and the instanton number is identified with the baryon number. Then, in this confined phase, we can examine the nuclear matter through the gas of instantons.

\section{A. Coordinate $z$ and embedding of the instanton}

In proceeding the analysis mentioned above, it is necessary to choose the coordinate $z$ carefully. For example, we may choose the four coordinates to make the instanton as

$$
\left(\xi^{0}, \xi^{1}, \xi^{2}, \xi^{3}, \xi^{4}\right)=\left(x^{0}, x^{1}, x^{2}, x^{3}, r\right),
$$

namely as $z=r$. In this case, using (2.13) and (3.1), we have

$$
\begin{aligned}
S_{\mathrm{V}} & =\int d^{6} \xi \sqrt{-g} \mathcal{L}_{\mathrm{V}}, \\
& =\int d x^{0} d x^{1} d x^{2} d x^{3} d w d r r^{4}\left(-\frac{3}{2} Q^{2}\left[\frac{1}{r^{4}}+f(r)\right]\right) \\
& =\int d x^{0} d w d r r^{4}\left(-\frac{3}{2} 2 N_{\mathrm{I}} \bar{q}^{2}\left[\frac{1}{r^{4}}+f(r)\right]\right) \\
& =N_{\mathrm{I}} \int d x^{0} d w d r r^{4}\left(-\frac{3}{2} \frac{\pi^{2} \rho^{4}}{\left(r^{2}+\rho^{2}\right)^{5 / 2}}\left[\frac{1}{r^{4}}+f(r)\right]\right) .
\end{aligned}
$$

Here we find a logarithmic divergence in the integration over $r$. It is understood as follows:

$$
\begin{aligned}
S_{\mathrm{V}} & \propto \int_{r_{0}}^{\infty} d r r^{4}\left(-\frac{3}{2} \frac{\pi^{2} \rho^{4}}{\left(r^{2}+\rho^{2}\right)^{5 / 2}}\left[\frac{1}{r^{4}}+f(r)\right]\right) \\
& \underset{r \rightarrow \infty}{\sim} \int^{\infty} d r\left(-\frac{3}{2} \frac{\pi^{2} \rho^{4}}{r}\right) \sim-\frac{3}{2} \pi^{2} \rho^{4} \log (\infty) .
\end{aligned}
$$

This fact implies that we should reset $z$ to another coordinate which leads to a finite energy density of the instanton.

A clue to finding such coordinate is seen in the top-down model of D4/D8 model $[2,14,15]$. In the case of the topdown model, D8-flavor brane is embedded in a set of special coordinates, which provide a flat space of the $r-w$ plane near the point $r=r_{0}$ by avoiding a conical singularity. This is performed in the present case by changing the coordinates from $(r, w)$ to $(z, \theta)$ as,

$$
\begin{gathered}
r^{5}=r_{0}^{5}+r_{0}^{3} z^{2} \equiv k, \\
\theta=\frac{5}{2} w .
\end{gathered}
$$

In fact, in this case, the two dimensional part of (3.1) is rewritten as

$$
\begin{aligned}
d s_{(2)}^{2} & =r^{2} f(r) d w^{2}+\frac{d r^{2}}{r^{2} f(r)} \\
& =\frac{4}{25}\left(\frac{r_{0}}{r}\right)^{3}\left(\frac{d z^{2}}{r^{2}}+z^{2} d \theta^{2}\right) .
\end{aligned}
$$

The second equation shows that the two dimensional metric is the polar coordinate of 2D flat space. Hereafter, we take $r_{0}=1$ for simplicity as in the top-down case. In this coordinate, the bulk $6 \mathrm{D}$ metric is written as

$d s^{2}=k^{2 / 5} \eta_{\mu \nu} d x^{\mu} d x^{\nu}+\frac{4}{25} k^{-3 / 5}\left(k^{-2 / 5} d z^{2}+z^{2} d \theta^{2}\right)$,

where $k=1+z^{2}$.

Here, noticing $\sqrt{-g}=(4 / 25) z$, the energy of the embedded instantons is given as

$$
\begin{gathered}
S_{\mathrm{V}}=\int d x^{0} d x^{1} d x^{2} d x^{3} d z \frac{4}{25} z\left(-\frac{3}{2} Q^{2}\left[k^{-4 / 5}+\frac{25}{4} k^{3 / 5}\right]\right) \\
=N_{\mathrm{I}} \int d x^{0} d z \frac{4}{25} z\left(-\frac{3}{2} \frac{\pi^{2} \rho^{4}}{\left(z^{2}+\rho^{2}\right)^{5 / 2}}\left[k^{-4 / 5}+\frac{25}{4} k^{3 / 5}\right]\right),
\end{gathered}
$$

where the factor $\int d \theta=2 \pi$ is absorbed to the gauge coupling constant. We can assure the finiteness of the $z$ integration of the above action. 


\section{B. CS term}

The baryon number is given by

$$
N_{\mathrm{B}}=\frac{1}{32 \pi^{2}} \int d^{3} x d z \epsilon^{m_{1} \cdots m_{4}} \operatorname{tr}\left(F_{m_{1} m_{2}} F_{m_{3} m_{4}}\right) .
$$

Then, the coupling of $A_{0}$ to the baryon number is given by the following CS term,

$$
\begin{aligned}
S_{\mathrm{CS}} & =\kappa_{\mathrm{CS}} \epsilon^{m_{1} \cdots m_{4}} \int d^{4} x d z A_{0} \operatorname{tr}\left(F_{m_{1} m_{2}} F_{m_{3} m_{4}}\right) \\
& =24 \kappa_{\mathrm{CS}} \int d^{4} x d z A_{0} Q^{2} \\
& =48 n \kappa_{\mathrm{CS}} \int d^{4} x d z A_{0} \bar{q}^{2},
\end{aligned}
$$

where

$$
n=\frac{N_{\mathrm{I}}}{V_{3}}, \quad V_{3}=\int d^{3} x=\int d x^{1} d x^{2} d x^{3} .
$$

\section{EFFECTIVE ACTION AND THE SIZE OF THE INSTANTON}

The matter action with embedded instantons is obtained here as

$$
\begin{aligned}
S_{\text {matter }}= & \int d^{6} \xi \sqrt{-g}\left(-\frac{1}{4} F^{2}-\frac{1}{4} \operatorname{tr} F_{S U(2)}^{2}\right)+S_{\mathrm{CS}} \\
= & \int d^{4} x d z\left(\frac{1}{2} z k^{3 / 5} A_{0}^{\prime 2}-n z \frac{12}{25} \bar{q}^{2}\left[k^{-4 / 5}+\frac{25}{4} k^{3 / 5}\right]\right. \\
& \left.+n n_{0} A_{0} \bar{q}^{2}\right)
\end{aligned}
$$

where $A_{0}^{\prime}=\partial_{z} A_{0}(z)$ and $n_{0}=48 \kappa_{\mathrm{CS}}$. In order to estimate this action, we solve the equation of motion of $A_{0}(z)$. It is obtained as

$$
-\partial_{z}\left(z k^{3 / 5} A_{0}^{\prime}\right)+n n_{0} \bar{q}^{2}=0 .
$$

Then we have

$$
z k^{3 / 5} A_{0}^{\prime}=\bar{d}=\frac{\pi^{2}}{6} n n_{0} \frac{2 z^{3}+3 z \rho^{2}}{\left(z^{2}+\rho^{2}\right)^{3 / 2}}+c .
$$

Here, $c$ denotes an integration constant of Eq. (4.2) over $z$. We take $c=0$ to set as $\bar{d}(z=0)=0$. Due to this condition, the matter action, $S_{\text {matter }}$ of (4.1), is written as

$$
\begin{aligned}
S_{\text {matter }}= & \int d^{4} x\left\{\mu \bar{Q}-\int d z\left(\frac{\bar{d}^{2}}{2 z k^{3 / 5}}\right.\right. \\
& \left.\left.+n z \frac{12}{25} \bar{q}^{2}\left[k^{-4 / 5}+\frac{25}{4} k^{3 / 5}\right]\right)\right\},
\end{aligned}
$$

where the chemical potential $\mu$ and the charge density $\bar{Q} \equiv \bar{d}(\infty)$ are given as

$$
\begin{aligned}
\mu=A_{0}(\infty) & =\int_{z_{1}}^{\infty} d z \frac{\bar{d}}{z k^{3 / 5}}+A_{0}\left(z_{1}\right) \\
& =\int_{z_{1}}^{\infty} d z \frac{\bar{d}}{z k^{3 / 5}} \\
\bar{Q} & =\bar{d}(\infty)=\frac{\pi^{2}}{3} n n_{0},
\end{aligned}
$$

where $z_{1}$ is an arbitrary positive value and $A_{0}\left(z_{1}\right)$ is set as

$$
A_{0}\left(z_{1}\right)=0,
$$

which is the boundary condition in solving the differential equation of $A_{0}(z)$, Eq. (4.3). Then the free energy density $\mathcal{E}$ of the instanton system is given as

$$
S_{\text {matter }}=-\int d^{5} \xi \mathcal{E}(\rho, \mu)
$$

$\mathcal{E}(\rho, \mu)=\int d z\left(\frac{\bar{d}^{2}}{2 z k^{3 / 5}}+n z \frac{12}{25} \bar{q}^{2}\left[k^{-4 / 5}+\frac{25}{4} k^{3 / 5}\right]\right)-\mu \bar{Q}$.

\section{A. Size of the instanton}

The free energy density $\mathcal{E}(\rho, \mu)$ in (4.9) is given as a function of $\rho$ for fixed $n, n_{0}, z_{1}$. Then we find the value of $\rho=\rho_{\min }$ where $\mathcal{E}(\rho, \mu(\rho))$ takes its minimum for a set of $\left(n, n_{0}, z_{1}\right)$; for this $\rho_{\min }, \mu$ and $\mathcal{E}(\rho, \mu)$ are determined. Repeating this procedure by changing only the value $n$ of the set $\left(n, n_{0}, z_{1}\right)$, we find different $\rho_{\min }$. As a result, we obtain the relationship between $\mu$ and $\mathcal{E}(\rho, \mu)$.

We notice that the results obtained in this way depend on the other two parameters $\left(n_{0}, z_{1}\right)$. We give a comment related to the parameter $z_{1}$. For $z_{1}=0$, we have a problem that the values of $\mu$ and $\mathcal{E}$ at $\rho=0$ are divergent,

$$
\begin{aligned}
&\left.\mu\right|_{\rho \rightarrow 0}=\int_{0}^{\infty} d z \frac{\bar{d}(\infty)}{z k^{3 / 5}} \\
& \underset{z \rightarrow 0}{\sim} \bar{d}(\infty) \int_{0} \frac{d z}{z} \rightarrow+\infty,
\end{aligned}
$$




$$
\mathcal{E}(\rho=0, \mu) \mid \underset{z \rightarrow 0}{\sim} \int_{0} d z\left(-\frac{\bar{d}(\infty)^{2}}{2 z k^{3 / 5}}\right) \rightarrow-\infty .
$$

In the present model these divergences are evaded by setting $z_{1}$, the lower bound of $z$, at a finite value. On the other hand, in the case of the top-down model [13], there are no such divergences. In fact, we can see that $\mu$ is finite even if $\rho$ is zero for $z_{1}=0$. Therefore, we expect the existence of some improvement of the present bottom-up model to resolve the above divergences. Here, this point remains as an open problem, and $z_{1}$ is introduced as a simple cutoff parameter for the above undesirable infrared divergences.

\section{B. EoS for nuclear matter}

By giving $n, n_{0}, z_{1}$, (which are the parameters of the present bottom-up model), $\mu(\rho)$ and $\mathcal{E}(\rho)$ are calculated according to the Eqs. (4.5) and (4.9) as functions of $\rho$. Then a minimum point of $\mathcal{E}(\rho)$ is found at $\rho=\rho_{\min }$. Thus, we can obtain $\mathcal{E}\left(\rho_{\min }\right)$ and $\mu\left(\rho_{\min }\right)$ for the given parameters $n, n_{0}, z_{1}$.

Repeating this procedure by changing only $n$ with $\left(n_{0}, z_{1}\right)$ fixed, we get the relationship of $\mu$ and $\mathcal{E}$. From this relationship we obtain the EoS of the instanton gas as mentioned above.

In Table I, we show a resultant example of such calculations for $n_{0}=1.5, z_{1}=0.1$. The regions of the calculations are restricted to the region for $\mu<1.7$ [2]. On the other hand, the pressure $p$, which is given by $p=-\mathcal{E}$, of the instanton gas is negative for $\mu<\mu_{c} \sim 0.17$. So in this region, the gas is in an undesirable phase as a nuclear matter considered here. ${ }^{1}$

Thus, we find that the stable nuclear matter exists in the region, $1.7>\mu>\mu_{c} \sim 0.17$. In this region of the nuclear matter, its dilute gas picture is reasonable since $v_{\min } \sim O\left(10^{-4}\right) \ll 1$, where $v_{\min }=\frac{4}{3} \pi \rho_{\min }^{3} n$ indicates the volume which is occupied by instantons in a unit 3D volume.

Here we proceed with the analysis, and we can arrive at the following approximate formula

$$
p=a \mu\left(\mu-\mu_{c}\right)
$$

where $a=0.13$ and $\mu_{c}=0.17$ (see Fig. 2). Then using (4.12), the energy density is given at $T=0$ as [18]

$$
\epsilon=\mu q-p=\mu \frac{\partial p}{\partial \mu}-p=a \mu^{2}
$$

and the speed of sound is obtained as

\footnotetext{
${ }^{1}$ The negative pressure state might be constructed under a delicate balance of two kinds nuclear forces [27,28]. Although this point is very interesting, we will discuss it in future work.
}

TABLE I. EoS of the nuclear system at low temperature for $n_{0}=1.5, z_{1}=0.1$, and $v_{\min }=\frac{4}{3} \pi \rho_{\min }^{3} n$.

\begin{tabular}{lllcc}
\hline \hline$n$ & $\mu\left(\rho_{\min }\right)$ & $\mathcal{E}\left(\rho_{\min }\right)$ & $\rho_{\min }$ & $v_{\min }$ \\
\hline 0.005 & 0.0688 & 0.00031 & 0.01 & $2.09 \times 10^{-8}$ \\
0.01 & 0.138 & 0.0000393 & 0.02 & $3.35 \times 10^{-7}$ \\
0.015 & 0.209 & -0.00022 & 0.03 & $1.70 \times 10^{-6}$ \\
0.02 & 0.275 & -0.00178 & 0.04 & $5.36 \times 10^{-6}$ \\
0.03 & 0.411 & -0.00829 & 0.06 & $2.75 \times 10^{-5}$ \\
0.05 & 0.68 & -0.0368 & 0.09 & $1.52 \times 10^{-4}$ \\
0.1 & 1.335 & -0.2075 & 0.13 & $9.2 \times 10^{-4}$ \\
\hline \hline
\end{tabular}

$$
C_{s}^{2}=\frac{\partial p}{\partial \epsilon}=\frac{\partial p / \partial \mu}{\partial \epsilon / \partial \mu}=1-\frac{\mu_{c}}{2 \mu}
$$

This leads to the following bound of $C_{s}^{2}$,

$$
\frac{1}{2}<C_{s}^{2}<1
$$

We notice that the lower bound $1 / 2$ is fairly large compared to that of the ordinary nuclear matter. We should however notice that the constraint (4.15) for $C_{s}^{2}$ comes from the formula (4.12), which is an approximate formula available at small $\mu$. It is possible to improve (4.12) by adding correction terms of higher powers of $\mu$. For example, by adding a term like $\mu^{4}$, we can find a new formula, $p=a^{\prime} \mu\left(\mu-\mu_{c}^{\prime}\right)+b^{\prime} \mu^{4}$ with appropriate values for the parameters, $a^{\prime}, \mu_{c}^{\prime}, b^{\prime}$. In this case, we can see that the maximum value of $C_{s}$ is realized at $\mu$ smaller than 1 and its maximum value is suppressed from 1 , then it decreases to $C_{s}=1 / \sqrt{3}$ in the large $\mu$ region. However, as we can see, the correction term of $\mu^{4}$ becomes small in the small $\mu$ region. Then it is difficult to suppress the lower bound of $1 / 2$. We find a small value of sound speed in region of $0<C_{s}^{2}<1 / 2$ for $\mu<\mu_{c}$, where $\mu_{c}\left(\neq \mu_{c}^{\prime}\right)$ denotes the critical point and it satisfies $p\left(\mu_{c}\right)=0$. In this region of $\mu$, however, the pressure $p$ is negative, then the instanton gas cannot make a nucleon matter like a star. This implies that our model cannot cover the low-density part of the normal nuclear matter, in which the sound velocity decreases to zero from its upper bound $(1 / \sqrt{3})$. How to overcome this point remains as a future problem.

In this sense, the nuclear matter given here might be a special one. Our model is considered in a restricted region of density or $\mu, \mu_{c}<\mu<1.7$, where 1.7 denotes the transition point to the deconfined $\mathrm{RN}$ phase. Therefore, we continue our analysis by using a simple model with (4.12) and (4.13), and we arrive at the EoS of the nuclear matter given as the instanton gas. It is written as

$$
p=\epsilon-\sqrt{a \epsilon} \mu_{c} .
$$




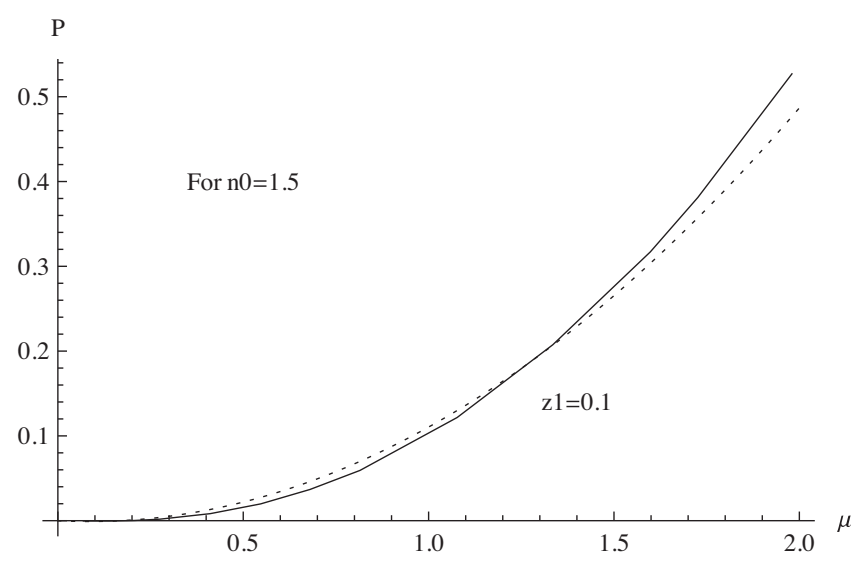

FIG. 2. $p=-\mathcal{E}(\mu)$ versus $\mu$ at stable instanton size $\rho_{\text {min }}$ near the phase transition point $\mu_{c}=0.17$. The solid curve denotes the numerical calculations, and the dotted curve represents $p=0.13 \mu\left(\mu-\mu_{c}\right)$.

\section{Alternative approach to find the EoS}

In the present scheme, the free energy density (4.9) takes $n, n_{0}, \rho$, and $z_{1}$ as independent variables. Among them, $n_{0}$ is an intrinsic parameter of the theory and $\rho$ is determined dynamically such that $\mathcal{E}$ takes its minimum value, while $z_{1}$ is set to be a plausible value for the moment. The free energy density is expanded in a power series of $n$,

$$
\mathcal{E}\left(n, \rho, n_{0}, z_{1}\right)=n c_{1}(\rho)-n^{2} n_{0}^{2} c_{2}\left(\rho, z_{1}\right),
$$

in which

$$
\begin{aligned}
c_{1}(\rho)= & \frac{12}{25} \frac{\pi^{2} \rho^{4}}{2} \int_{0}^{\infty} d z \frac{z}{\left(\rho^{2}+z^{2}\right)^{5 / 2}}\left[\left(1+z^{2}\right)^{-4 / 5}\right. \\
+ & \left.\frac{25}{4}\left(1+z^{2}\right)^{3 / 5}\right], \\
c_{2}\left(\rho, z_{1}\right)= & \left(\frac{\pi^{2}}{3}\right)^{2} \int_{0}^{\infty} d z \frac{1}{\left(1+z^{2}\right)^{3 / 5}}\left[\frac{1}{2} \frac{2 z^{2}+3 \rho^{2}}{\left(\rho^{2}+z^{2}\right)^{3 / 2}}\right. \\
& \left.-\frac{1}{8} \frac{z\left(2 z^{2}+3 \rho^{2}\right)^{2}}{\left(\rho^{2}+z^{2}\right)^{3}}\right] \\
& -\left(\frac{\pi^{2}}{3}\right)^{2} \int_{0}^{z_{1}} d z \frac{1}{\left(1+z^{2}\right)^{3 / 5}} \frac{1}{2} \frac{2 z^{2}+3 \rho^{2}}{\left(\rho^{2}+z^{2}\right)^{3 / 2}} .
\end{aligned}
$$

In order to change the independent variable from $n$ to $\mu$, we notice that $\mu$ can be written as

$$
\mu\left(n, \rho, n_{0}, z_{1}\right)=n n_{0} h\left(\rho, z_{1}\right)
$$

with

$$
h\left(\rho, z_{1}\right)=\frac{\pi^{2}}{6} \int_{z_{1}}^{\infty} d z \frac{1}{\left(1+z^{2}\right)^{3 / 5}} \frac{2 z^{2}+3 \rho^{2}}{\left(\rho^{2}+z^{2}\right)^{3 / 2}} .
$$

Now, eliminating $n$ by (4.20), the free energy density is rewritten as

$$
\mathcal{E}=-\frac{c_{2}\left(\rho, z_{1}\right)}{\left(h\left(\rho, z_{1}\right)\right)^{2}} \mu^{2}+\frac{c_{1}(\rho)}{n_{0} h\left(\rho, z_{1}\right)} \mu .
$$

From Eq. (4.22) one finds that the coefficients ( $a$ and $b$ ) and the critical chemical potential $\left(\mu_{c}\right)$ are given by

$$
a=\frac{c_{2}\left(\rho, z_{1}\right)}{h\left(\rho, z_{1}\right)^{2}}, \quad b=\frac{c_{1}(\rho)}{n_{0} h\left(\rho, z_{1}\right)}, \quad \mu_{c}=\frac{c_{1}(\rho) h\left(\rho, z_{1}\right)}{n_{0} c_{2}\left(\rho, z_{1}\right)} .
$$

Given $\mu, z_{1}$, and $n_{0}$, the minimum point of $\mathcal{E}$ (i.e., the maximum point of $p$ ) in the $\rho$ space can be sought numerically.

The minimum energy point depends on the variable which is taken to be fixed. Actually, two types of differential coefficients have different values as

$$
\left(\frac{\partial \mathcal{E}}{\partial \rho}\right)_{n}-\left(\frac{\partial \mathcal{E}}{\partial \rho}\right)_{\mu}=\left(\frac{\partial \mathcal{E}}{\partial \mu}\right)_{\rho}\left(\frac{\partial \mu}{\partial \rho}\right)_{n}=-\left(\frac{\partial \mathcal{E}}{\partial n}\right)_{\rho}\left(\frac{\partial n}{\partial \rho}\right)_{\mu}
$$

At the point $\rho=\rho_{\min }$ such that $(\partial \mathcal{E} / \partial \rho)_{n}=0$, it holds that

$$
\left(\frac{\partial \mathcal{E}}{\partial \rho}\right)_{\mu}=-\left(\frac{\partial \mathcal{E}}{\partial \mu}\right)_{\rho}\left(\frac{\partial \mu}{\partial \rho}\right)_{n}=(-b+2 a \mu) n n_{0} h_{\rho}\left(\rho, z_{1}\right) .
$$

In (4.25), one finds that

$$
\begin{aligned}
h_{\rho}\left(\rho, z_{1}\right) \equiv & \frac{\partial h\left(\rho, z_{1}\right)}{\partial \rho} \\
= & -\frac{\pi^{2}}{2\left(2 a_{0}\right)} \int_{z_{1}}^{\infty} d z \frac{1}{\left(1+z^{2}\right)^{3 / 5}} \frac{\rho^{3}}{\left(\rho^{2}+z^{2}\right)^{5 / 2}}<0, \\
& -b+2 a \mu=a\left(\mu-\mu_{c}\right)+a \mu>0,
\end{aligned}
$$

in the present situation, i.e., $a>0$ and $\mu>\mu_{c}$. Therefore, we conclude that

$$
\left(\frac{\partial \mathcal{E}}{\partial \rho}\right)_{\mu}<0 \quad\left(\text { at } \rho=\rho_{\min }\right) .
$$

This means that the minimum energy point along an $n$-fixed curve has much lower energy points towards the $\rho$-increasing direction with $\mu$ being kept constant. However, the problem is whether the minimum energy significantly depends on the fixed variable or not. 
To estimate the difference between the two types of minimum energies, one should continue numerical calculations on $\mathcal{E}$ from $\rho_{\min }$ by fixing $\mu$ and by increasing $\rho$ until one finds another minimum energy density $\mathcal{E}_{\text {min }}^{\prime}$. In the range of $0.015 \leq \rho \leq 0.1$ according to Table I, the calculations make the difference of the minimum energies explicit quantitatively such as $\left(\mathcal{E}_{\min }^{\prime}-\mathcal{E}_{\min }\right) / \mathcal{E}_{\min } \lesssim 0.028$.

We may consider that such differences do not affect the characteristic features of the EoS, and we again employ $n$ as the independent variable of $\mathcal{E}$ in the succeeding sections.

\section{TOV EQUATION AND $M-R$ RELATION OF NEUTRON STARS}

The TOV equations for a star with mass $m$, and $p$ as the radius $r$ in the star are given as

$$
\begin{gathered}
\frac{d p}{d r}=-G(\epsilon+p) \frac{m+4 \pi r^{3} p}{r(r-2 G m)}, \\
\frac{d m}{d r}=4 \pi r^{2} \epsilon
\end{gathered}
$$

Here, $G$ denotes the gravitational constant. They can be solved by using the EoS given in (4.16) with the boundary condition, $p(r=0)=p_{c}$ and $m(r=0)=0$. Solving these equations we obtain the mass of the star, $M=m(R)$, and its radius $R$, where $R$ is defined by $p(R)=0$.

In order to obtain the numerical results with dimensionful quantities, we must adjust the scale parameters for each dimensionful quantity $(\tilde{x})$. To do so, the above TOV equations are rewritten by using the dimensionless quantities as [19]

$$
\begin{gathered}
\frac{d \tilde{p}}{d \tilde{r}}=-B(\tilde{\epsilon}+\tilde{p}) \frac{\tilde{m}+4 \pi A \tilde{r}^{3} \tilde{p}}{\tilde{r}(\tilde{r}-2 B \tilde{m})}, \\
\frac{d \tilde{m}}{d \tilde{r}}=4 \pi A \tilde{r}^{2} \tilde{\epsilon},
\end{gathered}
$$

where

$$
A=\frac{r_{0}^{3} \epsilon_{0}}{m_{0}}, \quad B=\frac{G m_{0} \epsilon_{0}}{p_{0} r_{0}},
$$

and the various variables $(x)$ are replaced by the dimensionless quantities $(\tilde{x})$ by using its typical dimensionful value $x_{0}$ as $x=x_{0} \tilde{x}$. For example, we rewrite as $p=p_{0} \tilde{p}$.

Here we give a comment on the replacement, $\epsilon=\epsilon_{0} \tilde{\epsilon}$ and how $\epsilon_{0}$ is determined. In order to solve the TOV equation, $\epsilon(r)$ in the equation is replaced using Eq. (4.16) as a function of $p(r)$,

$$
\epsilon=\frac{a \mu_{c}^{2}}{4}\left(1+\sqrt{1+\frac{4 p}{a \mu_{c}^{2}}}\right)^{2} .
$$

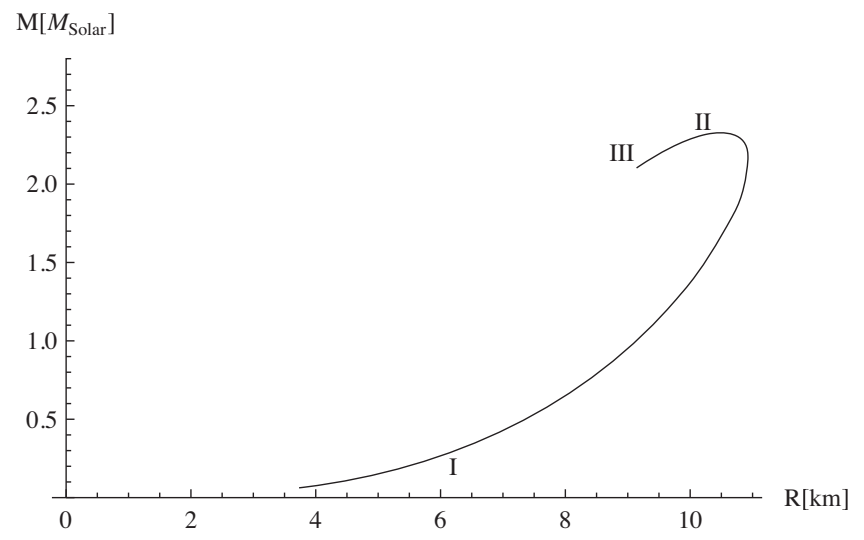

FIG. 3. Plot of the neutron mass $M(R)$ (in units of solar mass $\left.M_{\odot}\right)$ versus the radius $R$ for $p=\epsilon-\sqrt{a \epsilon} \mu_{c}$ [or $p=a \mu\left(\mu-\mu_{c}\right)$ ], $\mu_{c}=0.17$, and $a=0.13$.

Similar to the above, we introduce the dimensionless quantities as $a=a_{0} \tilde{a}, \mu_{c}=\mu_{0} \tilde{\mu}_{c}$, and

$$
\epsilon=\epsilon_{0} \tilde{\epsilon},
$$

by imposing reasonable conditions

$$
\epsilon_{0}=p_{0}=a_{0} \mu_{0}^{2}
$$

Then in Eqs. (5.3) and (5.4), we find

$$
\tilde{\epsilon}=\frac{\tilde{a} \tilde{\mu}_{c}^{2}}{4}\left(1+\sqrt{1+\frac{4 \tilde{p}}{\tilde{a} \tilde{\mu}_{c}^{2}}}\right)^{2},
$$

where we put the dimensionless number as $\tilde{a} \tilde{\mu}_{c}^{2}=$ $0.13 \times 0.17^{2}=3.76 \times 10^{-3}$.

Then the solutions of (5.3) and (5.4) with $A=B=1$ are equal to the one of (5.1) and (5.2) with $G=1$. Thus, the solution of the latter equations are translated to the one of the former ones as shown in Fig. 3.; it is given for $r_{0}=3.0 \mathrm{~km}$ in natural units. In this case, we find $m_{0}=2.03 M_{\odot}{ }^{2}$ and $\epsilon_{0}^{1 / 4}=0.896 \mathrm{GeV}$, which provides physical units to the quantities given in the Table 1. For example, the energy density of the center of the star, whose mass is about two solar masses, is given by $0.00242 \mathrm{GeV}^{4}$. This is about twice as large as the one of normal nuclear matter.

We can see that the radius and the mass increase with increasing $p_{c}$, the central value of the pressure. The resultant curve rotates anticlockwise toward the smaller radius in the large mass region. However from the point II to the point III in Fig. 3, the state would be unstable since $\partial M\left(\epsilon_{c}\right) / \partial \epsilon_{c}<0$ [19]. On the other hand, in the region from I to II, we can see the behavior $\partial M\left(\epsilon_{c}\right) / \partial \epsilon_{c}>0$.

\footnotetext{
${ }^{2}$ The symbol $M_{\odot}$ denotes the solar mass.
} 
Thus, the results given here indicate the possibility of the existence of a star with $(M, R) \sim\left(2 M_{\odot}, 10 \mathrm{~km}\right)$.

\section{COMPARISON WITH OBSERVATIONAL DATA}

There are some important observational data which can constrain the theoretical EoS. We now have at least three important constraints for the $M-R$ relation;

(1) Two solar mass $\left(2 M_{\odot}\right)$ neutron star observation from the Shapiro-delay measurement $[29,30]$.

(2) The radius constraint from GW170817 via the gravitational wave observation. The actual constraint is $9.0 \mathrm{~km}<R<13.6 \mathrm{~km}$ for $M=1.4 M_{\odot}$ [31-33].

(3) The upper bound of $M$ for cold spherical neutron stars which is estimated from no detection of relativistic optical counterpart in the analysis of GW170817. The actual limit is estimated as the range $2.15 M_{\odot}-2.26 M_{\odot}$ [34].

To support $2 M_{\odot}$ neutron stars in the theoretical calculation, we need a sufficiently stiff EoS which is closely related to the large value of the speed of sound. Then, the pressure of the matter in the inner neutron star core should be large. From the second constraint-the radius is relatively small in the moderate $M$ region-it requires that the pressure of the matter in the outer neutron core should be relatively small. It is interesting that our bottom-up model is quite simple, but our EoS can manifest the $2 M_{\odot}$ constraint and the restriction that the $M-R$ curve must go through $9.0<R<13.6$ around $M \sim 1.4 M_{\odot}$. However, a too rigid EoS is not preferable because of the third constraint. Unfortunately, the maximum mass $M_{\max }$ overshoots the $2.15 M_{\odot}-2.26 M_{\odot}$ constraint, but we can expect that there are some phase transitions at high density such as the chiral, deconfined, and color superconducting phase transitions which we do not consider in this study. Then, the EoS can be softer than the present one at high density and thus $M_{\max }$ should be smaller than the present value. Particularly, the EoS becomes soft if there is a first-order phase transition; for example, see Ref. [35].

It should be noted that the above constrains can restrict the large and moderate $M$ regions, but the lower $M$ region is not restricted so much. Of course, there are several nuclear theoretical EoS and thus we can qualitatively discuss the lower $M$ region where the low density part of the EoS dominates the $M-R$ curve. With our EoS, the $M-R$ curve flows down to the bottom left, but several $M-R$ curves with different EoSs flow down to the bottom right. This result may be induced from the fact that our $C_{s}^{2}$ cannot be less than $1 / 2$ even in a sufficiently low-density region. Such behavior is similar to the $M-R$ curves obtained with EoS proposed in Ref. [36]; the actual behavior of the curves can be seen in Refs. [37,38] denoted as "SQM1-3" and these EoS which contain the quark matter make self-bounded neutron stars which do not have minimum masses. The tendency of the $M-R$ curves in the small $M$ region in our model may be modified when we suitably introduce "interactions" to the present model because we employ the dilute instanton gas approximation; the baryonic contribution can be expected to have relatively strong effects on the EoS at low density. In addition, we here use the dilute gas of the instanton of the $S U(2)$ gauge field and thus the number of flavors is considered as two; the system contains the up and down quarks and thus there is no strange quark. This may explain the unclearness of the present $M-R$ curves because the EoS will be softer than the present one if hyperons appear in the system; since the core of the neutron star is very dense, the hyperon degree of freedom can join the game as the baryonic mode in addition to nucleons. This softening problem induced by the hyperons is the so-called hyperon puzzle which is a long standing problem in the study of the neutron stars with EoS; see Ref. [39] as an example. To discuss this problem in the present bottom-up holographic model, we must consider differences between the light quarks and the strange quark, interactions and bound states, more deeply. In the present study, we consider the simple symmetric nuclear matter because the difference between the flavors and the effects of the electrons are difficult to include in the model at present. Therefore, the inclusion of the effects such as the beta equilibrium and charge neutrality are left for our future work.

\section{SUMMARY AND DISCUSSIONS}

In this paper, we have studied cold nuclear matter and its equation of state based on the six-dimensional holographic model, which was investigated previously to realize the color superconducting phase of QCD. The nuclear matter was introduced as a dilute gas of deformed instantons in the AdS soliton background. The instantons are electric-charge neutral and they are made up of the $S U(2)$ gauge fields; the action includes the Chern-Simons term as well as the kinetic term. Owing to the energy balance between these two terms in the action, the size of the instanton can be determined for fixed parameters of the model. As the result, the EoS for nuclear matter, i.e., pressure as a function of energy density, is obtained. Through the evaluation of the instanton size, it turns out that the number of instantons in a unit volume is very small so that our dilute gas picture is consistent. Furthermore, there exists a critical value for the chemical potential below which the pressure becomes negative. The EoS obtained here is very stiff because the speed of sound $C_{s}^{2}>\frac{1}{2}$. As discussed in the Sec. IV B, this constraint is reduced to our simplified form of the pressure, $p(\mu)$. The lower bound $1 / 2$ can be suppressed when the functional form of $p(\mu)$ is modified.

Here, we however consider an EoS which is obtained from the simplified $p(\mu)$ to see the characteristic properties of our model. Then, we applied the simplified EoS to solve the Tolman-Oppenheimer-Volkov equations for a compact star numerically and found the $M-R$ relationship. The curve 
is somewhat similar to the one for strange quark matter in Refs. [36,38] and we provided a certain interpretation for that. Our result is compared with the observational data and it is seen that the maximum mass overshoots the current constraint since our EoS for the nuclear matter is very stiff. This might suggest that we have more phases such as quark matter, which softens the current EoS, than that of the nuclear matter. As we have shown in Sec. IV, our dilute gas picture is appropriate. There is a reason why we could achieve two solar mass neutron stars in spite of the diluteness of the instanton gas. In our approach, it might be possible that the mass of an instanton is very heavy compared with an ordinary nucleon, which is a specific property in holographic QCD since the nucleon mass is proportional to the number of colors $N_{c}$. So far we have no definite answer and this is a future issue.

In order to improve our current study, several ingredients are taken into account. One is to go beyond the dilute gas approximation of instantons. By doing this, our EoS is modified and eventually the $M-R$ curve might be changed so as to be more consistent with the observational data. The other is to study whether the system enjoys the phase transition from nuclear matter to (perhaps color superconducting) quark matter at higher baryon density. If such a phase transition is present, the EoS gets softened and the resultant $M-R$ curve could be modified. Furthermore, it will be interesting to extend our current study into the case with hyperon degrees of freedom. To this end, the holographic treatment of the heavy-light meson system (for instance, see [40]) must be instructive. These issues will be considered in the future.

\section{ACKNOWLEDGMENTS}

We are grateful D. Blaschke and A. Schmitt for their useful comments during the online conference "A Virtual Tribute to Quark Confinement and the Hadron Spectrum" (vConf2021). It is also a pleasure to thank Masayuki Matsuzaki for useful discussions on neutron stars and TOV equations.
[1] P. Basu, F. Nogueira, M. Rozali, J. B. Stang, and M. Van Raamsdonk, Towards a holographic model of color superconductivity, New J. Phys. 13, 055001 (2011).

[2] K. Ghoroku, K. Kashiwa, Y. Nakano, M. Tachibana, and F. Toyoda, Color superconductivity in holographic SYM theory, Phys. Rev. D 99, 106011 (2019).

[3] S. S. Gubser, Breaking an Abelian gauge symmetry near a black hole horizon, Phys. Rev. D 78, 065034 (2008).

[4] S. A. Hartnoll, C. P. Herzog, and G. T. Horowitz, Building an AdS/CFT Superconductor, Phys. Rev. Lett. 101, 031601 (2008).

[5] S. A. Hartnoll, C. P. Herzog, and G. T. Horowitz, Holographic superconductors, J. High Energy Phys. 12 (2008) 015.

[6] T. Nishioka, S. Ryu, and T. Takayanagi, Holographic superconductor/insulator transition at zero temperature, J. High Energy Phys. 03 (2010) 131.

[7] N. Iqbal, H. Liu, M. Mezei et al., Quantum phase transitions in holographic models of magnetism and superconductors, Phys. Rev. D 82, 045002 (2010).

[8] A. Chamblin, R. Emparan, C. V. Johnson, and R. C. Myers, Charged AdS black holes and catastrophic holography, Phys. Rev. D 60, 064018 (1999).

[9] K. B. Fadafan, J. C. Rojas, and N. Evans, A holographic description of colour superconductivity, Phys. Rev. D 98, 066010 (2018).

[10] C. H. Nam, A more realistic holographic model of color superconductivity with higher derivative corrections, Phys. Rev. D 104, 046006 (2021).

[11] O. Bergman, G. Lifschytz, and M. Lippert, Holographic nuclear physics, J. High Energy Phys. 11 (2007) 056.
[12] M. Rozali, H.-H. Shieh, M. Van Raamsdonk, and J. Wu, Cold nuclear matter In holographic QCD, J. High Energy Phys. 01 (2008) 053.

[13] K. Ghoroku, K. Kubo, M. Tachibana, T. Taminato, and F. Toyoda, Holographic cold nuclear matter as dilute instanton gas, Phys. Rev. D 87, 066006 (2013).

[14] T. Sakai and S. Sugimoto, Low energy hadron physics in holographic QCD, Prog. Theor. Phys. 113, 843 (2005).

[15] H. Hata, T. Sakai, S. Sugimoto, and S. Yamato, Baryons from instantons in holographic QCD, Prog. Theor. Phys. 117, 1157 (2007).

[16] F. Preis and A. Schmitt, Layers of deformed instantons in holographic baryon matter, J. High Energy Phys. 07 (2016) 001.

[17] C. Hoyos, D. R. Fernandez, N. Jokela, and A. Vuorinen, Holographic Quark Matter and Neutron Stars, Phys. Rev. Lett. 117, 032501 (2016).

[18] K. B. Fadafan, J. C. Rojas, and N. Evans, Deconfined, massive quark phase at high density and compact stars: A holographic study, Phys. Rev. D 101, 126005 (2020).

[19] K. Bitaghsir Fadafan, J. C. Rojas, and N. Evans, Holographic quark matter with colour superconductivity and a stiff equation of state for compact stars, Phys. Rev. D 103, 026012 (2021).

[20] K. Nawa, H. Suganuma, and T. Kojo, Brane-induced Skyrmion on $S^{3}$ : Baryonic matter in holographic QCD, Phys. Rev. D 79, 026005 (2009).

[21] M. Rho, S. J. Sin, and I. Zahed, Dense QCD: A holographic dyonic salt, Phys. Lett. B 689, 23 (2010).

[22] F. Preis and A. Schmitt, Layers of deformed instantons in holographic baryonic matter, J. High Energy Phys. 07 (2016) 001. 
[23] M. Elliot-Ripley, P. Sutcliffe, and M. Zamaklar, Phases of kinky holographic nuclear matter, J. High Energy Phys. 10 (2016) 088.

[24] T. Ishii, M. Järvinen, and G. Nijs, Cool baryon and quark matter in holographic QCD, J. High Energy Phys. 07 (2019) 003.

[25] T. Nakas and K. S. Rigatos, Dependence of inclusive jet production on the anti-kT distance parameter in pp collisions at $\sqrt{S}=13 \mathrm{TeV}$, J. High Energy Phys. 12 (2020) 157.

[26] E. Witten, Anti-de Sitter space, thermal phase transition, and confinement in gauge theories, Adv. Theor. Math. Phys. 2, 505 (1998).

[27] M. Berthelot, Sur quelques phenomenes de dilatation force des liquides, Ann. Chim. Phys. 30, 332 (1850).

[28] T. D. Cohen, S. Lawrence, and Y. Yamauchi, The thermodynamics of large-N QCD and the nature of metastable phases, Phys. Rev. C 102, 065206 (2020).

[29] P. Demorest, T. Pennucci, S. Ransom, M. Roberts, and J. Hessels, Shapiro delay measurement of a two solar mass neutron star, Nature (London) 467, 1081 (2010).

[30] J. Antoniadis, P. C. C. Freire, N. Wex, T. M. Tauris, R. S. Lynch, M. H. van Kerkwijk, M. Kramer, C. Bassa, V. S. Dhillon, and T. Driebe et al., A massive pulsar in a compact relativistic binary, Science 340, 6131 (2013).

[31] E. Annala, T. Gorda, A. Kurkela, and A. Vuorinen, Gravitational-Wave Constraints on the Neutron-Star-Matter Equation of State, Phys. Rev. Lett. 120, 172703 (2018).
[32] S. De, D. Finstad, J. M. Lattimer, D. A. Brown, E. Berger, and C.M. Biwer, Tidal Deformabilities and Radii of Neutron Stars from the Observation of GW170817, Phys. Rev. Lett. 121, 091102 (2018); Erratum, Phys. Rev. Lett. 121, 259902 (2018).

[33] I. Tews, J. Margueron, and S. Reddy, Critical examination of constraints on the equation of state of dense matter obtained from GW170817, Phys. Rev. C 98, 045804 (2018).

[34] M. Shibata, S. Fujibayashi, K. Hotokezaka, K. Kiuchi, K. Kyutoku, Y. Sekiguchi, and M. Tanaka, Modeling GW170817 based on numerical relativity and its implications, Phys. Rev. D 96, 123012 (2017).

[35] T. Kojo, QCD equations of state and speed of sound in neutron stars, AAPPS Bull. 31, 11 (2021).

[36] M. Prakash, J. R. Cooke, and J. M. Lattimer, Quarkhadron phase transition in protoneutron stars, Phys. Rev. D 52, 661 (1995).

[37] J. M. Lattimer and M. Prakash, Neutron star structure and the equation of state, Astrophys. J. 550, 426 (2001).

[38] F. Ozel, D. Psaltis, T. Guver, G. Baym, C. Heinke, and S. Guillot, The dense matter equation of state from neutron star radius and mass measurements, Astrophys. J. 820, 28 (2016).

[39] W. Weise, Dense baryonic matter and strangeness in neutron stars, J. Phys. Soc. Jpn. Conf. Proc. 26, 011002 (2019).

[40] J. Erdmenger, K. Ghoroku, and I. Kirsch, Holographic heavy-light mesons from non-Abelian DBI, J. High Energy Phys. 09 (2007) 111. 